\title{
Structural Health Monitoring of an Offshore Wind Turbine Tower Using iFEM Methodology
}

\author{
Mingyang $\mathrm{Li}^{1}$, Adnan $\mathrm{Kefal}^{2,3,4,5}$, Erkan Oterkus ${ }^{1, *}$, and Selda Oterkus ${ }^{1}$ \\ ${ }^{1}$ Department of Naval Architecture, Ocean and Marine Engineering, University of Strathclyde, \\ Glasgow, United Kingdom \\ ${ }^{2}$ Faculty of Engineering and Natural Sciences, Sabanci University, Tuzla, Istanbul 34956, Turkey \\ ${ }^{3}$ Integrated Manufacturing Technologies Research and Application Center, Sabanci University, Tuzla, \\ 34956, Istanbul, Turkey \\ ${ }^{4}$ Composite Technologies Center of Excellence, Istanbul Technology Development Zone, Sabanci \\ University-Kordsa Global, Pendik, Istanbul 34906, Turkey \\ ${ }^{5}$ Faculty of Naval Architecture and Ocean Engineering, Istanbul Technical University, Maslak-Sariyer \\ 34469, Istanbul, Turkey.
}

Abstract: With the increasing popularity of wind energy, offshore wind turbines (OWTs) are currently experiencing rapid development. The tower is one of the most significant components of the OWT. However, the tower will not only stand its own weight and weight of the top structure, but also be surrounded by harsh wave and wind loading conditions. Therefore, it is necessary to apply a structural health monitoring (SHM) system to monitor the health condition of the OWT towers in realtime. In this study, inverse Finite Element Method (iFEM) is applied to monitor the tower of an OWT under both static and dynamic loading conditions. The total displacements and von Mises stresses obtained from iFEM analysis are compared against reference results and optimum sensor locations are determined.

Keywords: Offshore Wind Turbine; iFEM; Tower; Structural Health Monitoring

\section{Introduction}

In order to control climate change and decrease the emission of greenhouse gases, governments and industry are trying to replace fossil fuel with renewable energy. Among different sources of renewable energy, wind energy, one of the most environmental-friendly energy without any pollution to the environment, is playing an increasingly important role. Since the technology of utilizing wind energy is wellestablished compared to other types of renewable energy sources and the cost of electricity generated from wind is relatively inexpensive, wind energy is expected to experience fast and tremendous development during the following decades [1,2]. Currently, a large number of wind turbines have been installed and majority of them 
are located onshore [3]. However, due to the fact that suitable locations with ideal wind conditions are becoming limited and onshore wind turbines also cause some visual and noise problems, OWTs are becoming a popular option in the industry. OWTs placed at remote positions along the coast are usually surrounded by strong and stable wind resources and they can generate more electricity than onshore wind turbines. With the improvement of technology, OWTs are tending to move into deeper water and the capacity of the turbines becomes larger.

The OWT is a kind of unmanned and remote-controlled structure [4]. However, OWTs usually suffer from unpredictable and harsh environments. Apart from common loads like strong wind and severe waves, OWTs are also facing some shortterm extreme loadings caused by storms, heavy snow, and even earthquakes [4]. Besides, OWTs are also bearing the thickness reduction caused by corrosion and sometimes marine accidents like collision. The structural damage of each component of the OWTs may result in catastrophic collapse of the whole structure. In order to gain more energy, the size of the OWTs is becoming larger and complex. It is undoubted that the maintenance of OWTs is more difficult and expensive. Additionally, OWTs are usually difficult to be accessible for maintenance. Large lifting equipment and vessels are usually required which will increase the expense of the repairing process. There may also be a period of shut down of the OWTs caused by the failure and delay of the repair. All of these factors will lead to maintanance cost of OWTs roughly over twice of the onshore wind turbines [5]. It is estimated that the cost of maintenance and operation may occupy $10 \%-15 \%$ of the general income of the OWTs [6]. When it comes to the end of the OWTs' lifetime, this percentage may rise to $35 \%$ of the whole OWT project cost [1]. For the sake of ensuring the safety conditions of the OWTs and decreasing the cost of the maintenance, accurate and robust SHM systems are quite necessary to be installed on OWTs. For the typical SHM process, initially, the sensing systems will be installed on the structure at proper locations. Then, the sensing systems will collect the required real-time data from the structure and report the data to the SHM systems. Finally, utilizing the collected data, the SHM systems can obtain the global or local state of the monitored structure [7]. According to Gherlone et al. [8], a good SHM system should easily treat the complicated structures and their boundary conditions. Moreover, the loading conditions, material properties and even some inherent errors (cannot be avoided 
during the process of measuring data) should not affect the stability and accuracy of the system. In addition, the SHM system must be fast enough to perform real-time monitoring process. [8] With the help of the SHM system, unusual behaviors, such as unhealthy conditions and structural failure, of the structure can be accurately detected. Furthermore, additional management including inspection, maintenance, and repair of the structure can also be enhanced under the guidance of SHM systems [6]. The process of maintenance can be scheduled in a more orderly manner, which will reduce the unnecessary inspection and repair. For an OWT, SHM systems can contribute to generate stable and reliable electricity by decreasing the time of downtime. Therefore, productivity and financial benefit can be maximized. Even if there are no abnormal deformations or damage occurance, SHM can also be beneficial to investigate the behavior of the OWTs under complex loadings during the daily operation which can improve the design and manufacturing of the future OWTs [9].

For an OWT, Rotor Nacelle Assembly (RNA), tower and foundation are the main sections. Recently, there are studies which have been carried out to explore the application of SHM systems to these components. Weijtjens et al. [10] provide an SHM system based on both linear and non-linear regression models for the monopile foundation of an OWT in-service condition. The data generated by the SHM system is compared against the data collected in reality. The feasibility of this type of SHM system has been verified after the comparison. Mieloszyk et al. [3] selected an experimental method to test the practicality of applying the SHM systems to a tripod foundation by using Fiber Bragg Grating (FBG) sensors to collect the required data. Benefiting from their research, the SHM process for the foundations of the OWTs becomes more clear and practical. For the concrete foundations, Currie et al. [11] successfully monitored the displacements of the foundation of an onshore wind turbine by using a network of wireless sensors. Although there are some structural differences between the concrete foundations of the offshore and onshore turbines, this novel SHM approach can be an indicator for the research about the SHM system for the concrete foundations of the OWTs. Yi et al. [12] used strain gauges together with accelerometers and tiltmeters to form a SHM system which can be used for a long period of time for the jacket foundation. Devriendt et al. [13] and Rolfes et al. [14] both focused on health monitoring of the tower of the OWTs. Rolfes et al. explored the use of limited wireless sensors for damage detection. Differently, the 
dynamic behaviors of the tower were monitored by Devriendt [13]. Since the blades are one of the most critical components of RNA, Schroeder et al. [15] gave their attention to this component and they successfully utilized FBG sensors to achieve a SHM system for the estimation of the loading conditions on the blades of the OWTs. [15]

Different strategies and different methods have been investigated for the application of SHM systems to the OWTs. Usually, these studies focus on one specific component of the OWTs. Because of the complex topology of the OWTs and the complex loads caused by wind and wave, most of the current existing SHM systems do not concentrate on monitoring 3-dimensional full-field displacements and stresses of the OWTs. For the purpose of fulfilling this request, iFEM, developed by Tessler and Spangler [16-18], can be used as the state-of-the-art methodology for the SHM system of the OWTs. iFEM uses the strain data obtained by sensors to numerically restore the displacements, strains, and stresses of the structure in real-time. Based on the deformation information, the structural safety conditions can be judged. After comparing with other types of SHM methods, the advantages of iFEM can be summarized as: (1) iFEM analysis can take complex topology and boundary conditions of the structure into consideration, (2) the loading conditions together with material properties of the structure are not necessary for the determination of the displacement field, (3) iFEM can provide accurate and reliable results for the health condition in real-time regardless of whether the collected strain data has some errors or not and (4) if the locations of the sensors are logically and properly selected, it will not be necessary to install sensors to the entire structure which will drastically reduce the required number of sensors and make the SHM system more cost-effective [7, $19,20]$.

With the development of iFEM, different kinds of inverse finite elements have been generated (beam [21,22], frame [8,23], and shell [18,20,24,25]). For the OWTs, several shell elements would be more suitable to carry out the iFEM analysis. In the first place, the i3-RZT element was specifically developed for the iFEM analysis of structures made from composite materials [24,26]. The functionality and accuracy of the i3-RZT element have been proven by two laminated composite examples [24]. Lately, Kefal developed a curved inverse element with 8 nodes (iCS8) by 
incorporating the First-order Shear Deformation Theory (FSDT) to a shell element [25]. Despite iCS8 have some advantages when dealing with curved structures, the iQS4 element, also developed by Kefal et al. [20], can be easily used for iFEM analysis with high accuracy for shell structures and the application of the iQS4 element is proficient and well-established especially in marine and offshore fields. iQS4 element has been successfully applied by Kefal et al. [27-29] to perform SHM of a chemical tanker, a Panamax containership and a bulk carrier and these vessels are under different hydrodynamic loadings during the analysis. More importantly, the iQS4 element has shown its potential to detect the damage of the structure during the iFEM process [30]. Colombo et al. [31] continued this research by comparing the strains at single/multiple test locations. A plate with various cracks under a variety of loading conditions has been used to test the feasibility of this approach. By defining extra judgement criterion, iQS4 element is promising for damage prediction.

FBG technology should also be highlighted because FBG sensors are very suitable for SHM systems, especially for iFEM applications. FBG sensors have the advantages of less-weight and less-size compared against conventional strain gauges. Massive data can be collected by a single line which will effectively save the cost for data collection. Furthermore, FBG sensors have better corrosion resistance in marine environments than traditional sensors [32]. Currently, FBG sensors have already been used to obtain strain data for different components of the OWTs [15,33-35].

This study focuses on the application of the iQS4 element for the tower of a reference OWT [36]. The displacements and stresses of the tower under static and dynamic loading conditions are monitored. In Section 2, the theory of the iFEM-iQS4 element will be briefly described. Then, Section 3 will demonstrate the numerical procedure including the model generation, sensor selections and iFEM analysis of both static and dynamic cases. Finally, a summarized conclusion will be presented and future research aspects will be suggested. To the best of our knowledge, this is the first study using the iFEM-iQS4 element for the health monitoring of the tower of the OWTs. 


\section{Formulation of iQS4 element}

\section{1 iFEM-iQS4 element}

The first stage of iFEM analysis is dividing the whole structure into a reasonable number of iQS4 elements. The iQS4 element is defined on the basis of FSDT and has 4 nodes with 6 degrees of freedom (DOFs) per node. Three of them are displacements in the axial directions and the remaining three are the nodal rotations. Among the DOFs of the iQS4 element, drilling rotation is specially considered. Hence, the singularity problem can be easily solved and the shear locking phenomenon can also be minimized which makes the iQS4 element quite suitable for modeling complicated shell structures. The local coordinate system (LCS) of each iQS4 element is located at the central core of the element (Figure 1). The thickness of the element is evenly separated in the z-direction by the x-y plane of the LCS. The top layer of the element is represented by " $z=+h$ " and " $z=-h$ "stands for the bottom layer of the element. The shape functions as well as their derivatives, which are widely used in the Finite Element Method (FEM), can be used to establish the relationship between elemental strains and nodal displacements. There are three strain-displacement matrices, which are defined as $\mathbf{B}_{m}, \mathbf{B}_{k}$, and $\mathbf{B}_{s}$, describing the membrane, bending, and transverseshear behaviors, respectively. The detailed expressions of the shape functions of iQS4 element and their usage to form these strain-displacement matrices can be found in Kefal et al. [20]. Analytical section strain components in each element can be numerically calculated by the following functions:

$$
\begin{array}{r}
\mathbf{e}=\mathbf{B}_{m} \mathbf{u}_{e} \\
\mathbf{k}=\mathbf{B}_{k} \mathbf{u}_{e} \\
\boldsymbol{\gamma}=\mathbf{B}_{s} \mathbf{u}_{e}
\end{array}
$$

where $\mathbf{e}, \mathbf{k}$, and $\boldsymbol{\gamma}$ are the membrane, bending, and transverse-shear section strains and the $\mathbf{u}_{e}$ vector contains the arrays of the 6 DOFs at each node $(i=1-4)$ depicted in Figure 1(b). 


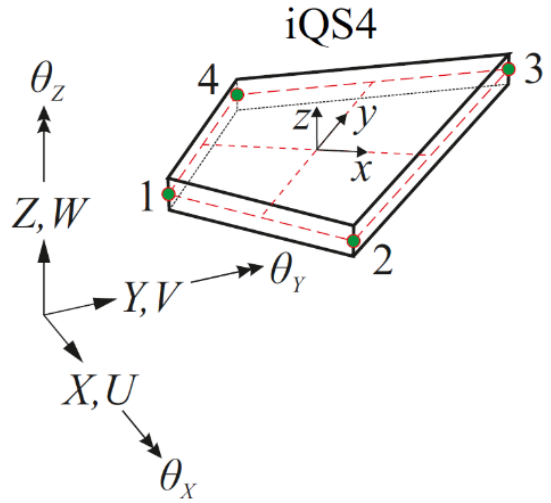

(a)

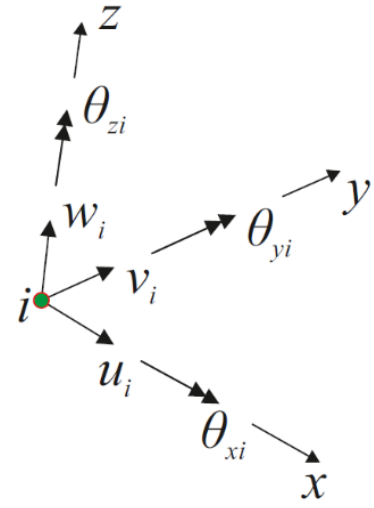

(b)

Figure 1. (a) iQS4 element with local coordinate system (LCS) $(x, y, z)$ at the central plane and global coordinate system (GCS) $(X, Y, Z)$ of the structure (b) the total 6 DOFs in LCS [20]

\subsection{Strain collection by sensors}

Strain data collection is one of the most important processes of iFEM analysis. FBG sensors are recommended and preferred for fulfilling this purpose because the required strains can be obtained by just several lines of FBG sensors. After the discretization of the structure, the sensors will be installed on both sides of the iQS4 element (Figure 2). For the current analysis, sensors are placed at the centre of each iQS4 element and there are only two sensors attached at the top and bottom surfaces. The symbol "+" represents the strains obtained from the top surface of the element and "-" represents the strains gathered from the bottom side. The experimental strains of membrane and curvatures can be calculated by the equations shown below:

$$
\begin{aligned}
& \mathbf{e}^{*}=\frac{1}{2}\left\{\begin{array}{l}
\varepsilon_{x x}^{+}+\varepsilon_{x x}^{-} \\
\varepsilon_{y y}^{+}+\varepsilon_{y y}^{-} \\
\gamma_{x y}^{+}+\gamma_{x y}^{-}
\end{array}\right\} \\
& \mathbf{k}^{*}=\frac{1}{2 h}\left\{\begin{array}{l}
\varepsilon_{x x}^{+}-\varepsilon_{x x}^{-} \\
\varepsilon_{y y}^{+}-\varepsilon_{y y}^{-} \\
\gamma_{x y}^{+}-\gamma_{x y}^{-}
\end{array}\right\}
\end{aligned}
$$

It should be mentioned that transverse shear strains cannot be obtained by the sensors. Since the plane stress condition can be utilized as a simplification for most of the offshore structural problems, which means that the shell structure can be regarded as "thin shell", the transverse shear strains are much smaller than the remaining two. So 
in such cases, the transverse strains can be ignored without any big impact on the accuracy.

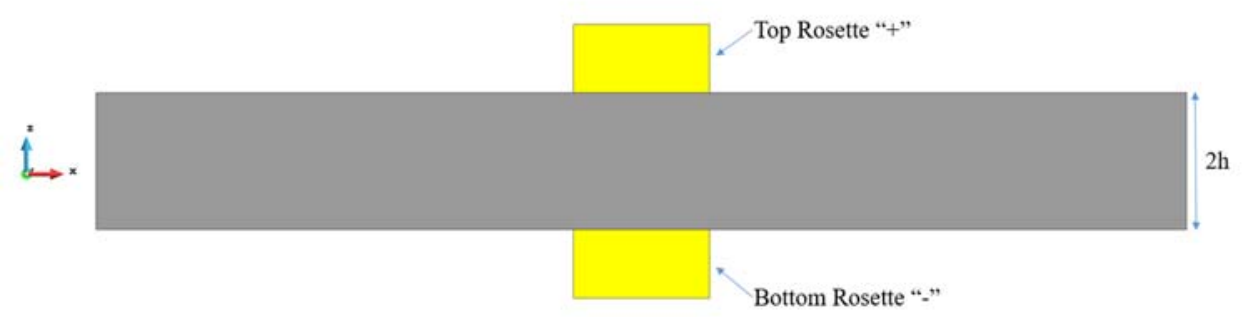

Figure 2. Strain collection for iQS4 element using FBG sensors

\subsection{Basic equations of iQS4 elements}

The basic idea of iFEM analysis is to minimize the difference between experimental strains and analytical strains for each iQS4 element. The differences between the numerically calculated $\mathbf{e}, \mathbf{k}$, and $\boldsymbol{\gamma}$ against the experimental values can be defined by using the functional, $\boldsymbol{\varphi}_{e}$. The expression of $\boldsymbol{\varphi}_{e}$ can be written as:

$\boldsymbol{\varphi}_{e}=\omega_{m}\left\|\mathbf{e}-\mathbf{e}^{*}\right\|^{2}+\omega_{k}\left\|\mathbf{k}-\mathbf{k}^{*}\right\|^{2}+\omega_{s}\left\|\gamma-\gamma^{*}\right\|^{2}$

where $\omega_{m}, \omega_{k}$, and $\omega_{s}$ are the weighting constants. If the experimental strains are available, the weighting constants can be equal to 1 . However, if the strain data cannot be measured by the sensors, it is suggested that a small number like $10^{-4}$ or $10^{-5}$ can be chosen as the weighting constants. By doing so, the interpolation connectivity of the iQS4 element can still be guaranteed.

By differentiating $\boldsymbol{\varphi}_{e}$ against the unknown displacements, the minimization of the differences between experimental and numerical strains can be achieved. A matrix system (similar to FEM) can be obtained as a consequence of differentiation

$\frac{\partial \boldsymbol{\varphi}_{e}}{\partial \mathbf{u}_{e}}=\mathbf{k}_{e} \mathbf{u}_{e}-\mathbf{f}_{e}=0 \Rightarrow \mathbf{k}_{e} \mathbf{u}_{e}=\mathbf{f}_{e}$

where $\mathbf{k}_{e}$ is a matrix formed by strain-dispalcement matrices and weighting functions.

Once the $\mathbf{k}_{e}$ matrix is established, unless the geometry or the boundary condition changes, there is no need to change it during the iFEM analysis and this will contribute to the reduction of the run time which makes iFEM analysis suitable for real-time monitoring. The $\mathbf{f}_{e}$ is an array formed by the collected strain data. Finally, 
$\mathbf{u}_{e}$ is the desired nodal displacements. After transforming from LCS to GCS, applying boundary conditions and assembling the global system of equations, fullfield displacements can be reckoned.

\subsection{Post-processing for iFEM analysis}

The health condition of the structure can be assessed with the help of 6 regenerated global displacements and rotations $\left(U, V, W, \theta_{x}, \theta_{y}, \theta_{z}\right)$. Moreover, by means of additional parameters, such as total displacements, total rotations, and von Mises strain/stress, the health condition can be determined with a step further. Total displacements $\left(U_{T}\right)$ and von Mises stress $\left(\sigma_{v m}\right)$ are commonly used. The total displacements can be forthrightly calculated as:

$U_{T}=\sqrt{U^{2}+V^{2}+W^{2}}$

Three independent displacements can be replaced by $U_{T}$ and the extreme values combining with the plots of $U_{T}$ can give a comprehensive but brief illustration of the deformation condition of the structure.

The strains of each iQS4 element can also be calculated after obtaining the nodal displacements. Previously introduced $\mathbf{B}_{m}, \mathbf{B}_{k}$, and $\mathbf{B}_{s}$ matrices can be used to together with prediceted $\mathbf{u}_{e}$ vectors to establish the individual strain components. Then, the stress distribution of the iQS4 element can be computed utilizing the constutive relations of the plane-stress condition. Finally, once the normal and shear stresses components are obtained, they can be subsequently used along with the von Mises stress criterion to make a reasonable failure assessments and identification of structurally unhealthy condition.

\section{Numerical results}

\subsection{Model generation}

The geometry of the tower is determined based on the studies by Dagli et al. [36] and Gücüyen [37]. The tower is divided into 26 sections. Usually, each section should have its own thickness, height, and weight. But for simplification, the thickness of all of the sections is $30 \mathrm{~mm}$ and the height of each section is $2.5 \mathrm{~m}$. The total height of 
the tower is $65 \mathrm{~m}$ with $20 \mathrm{~m}$ height underwater. The maximum diameter of the tower is $4 \mathrm{~m}$ at the bottom and the diameter linearly decreases to $1.5 \mathrm{~m}$ at the top of the tower. The material of the tower is entirely considered as steel regardless of the steel grades. The Elastic Modulus is $210 \mathrm{GPa}$ and the Poisson's ratio is 0.3 , respectively. The density of the steel is $7850 \mathrm{~kg} / \mathrm{m}^{3}$. However, in reality, there will be paints, cables, and ladders attached to the surface of the tower. Therefore, the weights of these components should also be included. It would be a reasonable way to transform the extra weights being divided by the volume of each section to the density of the shell element. Finally, the density of the structure is decided as $8500 \mathrm{~kg} / \mathrm{m}^{3}$. During the analysis, the weights of the structure itself will be applied by defining the gravitational acceleration. Additionally, for static analysis, the weight of the RNA, which is $83000 \mathrm{~kg}$, is acted on the top of the tower. The aerodynamic and hydrodynamic forces are further considered during the dynamic condition. The bottom of the tower will be fixed as the boundary condition for both static or dynamic conditions. The model of the tower is discretised by 8424 elements and 8460 nodes for FEM analysis (shown as Figure 3(a)). The results of FEM analysis are utilised for both reference solution and to generate "synthetic data" for the iFEM analysis. The mesh of the model in the iFEM process, which only contains 936 elements and 948 nodes (shown as Figure 3(b)), is much coarser than the FEM model. Therefore, for each iFEM element, there will be several FEM elements as the "synthetic" strain data provider. 


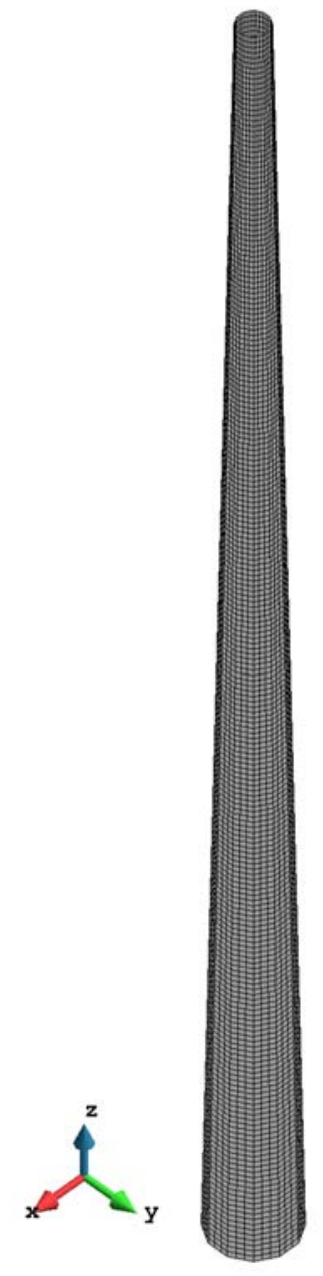

(a)

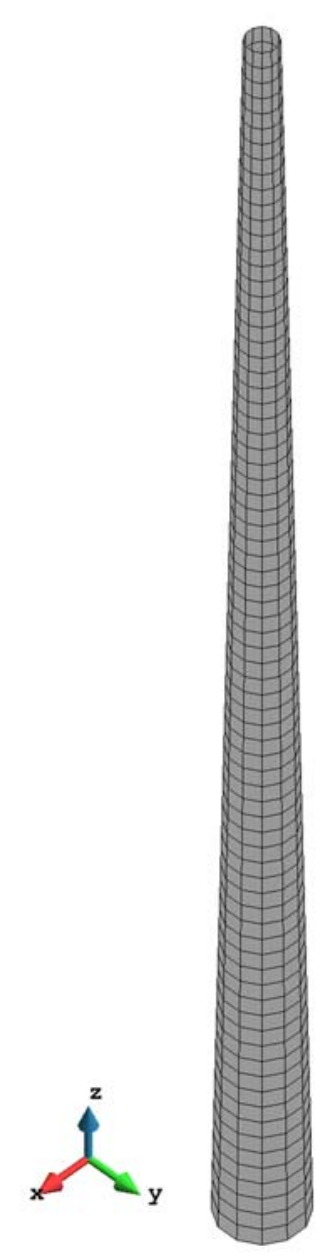

(b)

Figure 3. Mesh size (a) dense FEM mesh (b) coarse iFEM mesh

\subsection{Sensor selection and data evaluation}

In the first case, it is considered that all iFEM elements are instrumented with strain sensors. Therefore, the number of the sensors becomes 936x2. Although a large number of sensors is considered in this case, named as full-sensor condition, it can prove the accuracy and reliability of the iFEM process. Then, the number of elements with sensors is reduced to 328 to make it more practical. As can be seen from Figure 4(a), four longitudinal lines with sensors and two circumferential lines (one at the bottom and another at the top) with sensors are selected. The two ring lines of sensors are aimed to monitor the boundaries of the tower. It can not only reinforce the 
boundary condition for the analysis but also monitor the extreme deformations (especially in the z-direction) at the boundary. This distribution of sensors is used for both static and dynamic analysis. Because the directions of the wave and wind are not known in reality, symmetrical boundary conditions cannot be applied. Hence, the purpose of the four vertical lines of sensors is to monitor the health condition of the whole tower for any loading conditions. Besides, during the analysis, it was found that connecting the sensors at different parts of the structure together has the ability to improve the accuracy of the results and these four lines of sensors can strengthen the quality of the deformation estimation. With the help of FBG sensors, since these elements are located continuously, the strain data can be collected by just several lines of FBG sensors. However, with the increase of the sensor locations, although FBG technology can deal with collecting a great deal of data by just a single line, the efficiency of the FBG sensors will be significantly influenced and the process of separating the information to strains will also become complex. In order to relieve this problem, strain data evaluation would be an appropriate method and the missing strains can be estimated by the nearby strains. For the current analysis, after balancing the accurate level of the evaluation and the minimized number of sensors, each vertical line of sensors can be divided into four groups and different strategies can be utilized. Actually, for each section, there are 3 layers of elements in the vertical zdirection. First of all, for the sections around the boundary, all of the three elements are chosen. Secondly, for the sections around the free water surface, all elements will will also be selected because the strains around this region, especially in dynamic conditions, usually experience large variations. Then for the remaining underwater sections, the top and bottom elements are preferred to install the sensors. On the contrary, for the sections subjected to the aerodynamic loads, the sensors are only installed at the middle elements. The reason is that the strains of the sections above water is much more stable than the underwater parts. Eventually, the number of elements with sensors is decided as 176 and it only occupies about $2 \%$ of the total quantity of the FEM elements. It means that $6 \times 2$ lines of FBG sensors are enough to perform the iFEM analysis of the tower. This number can be further reduced, but it can have a negative influence on accuracy. 


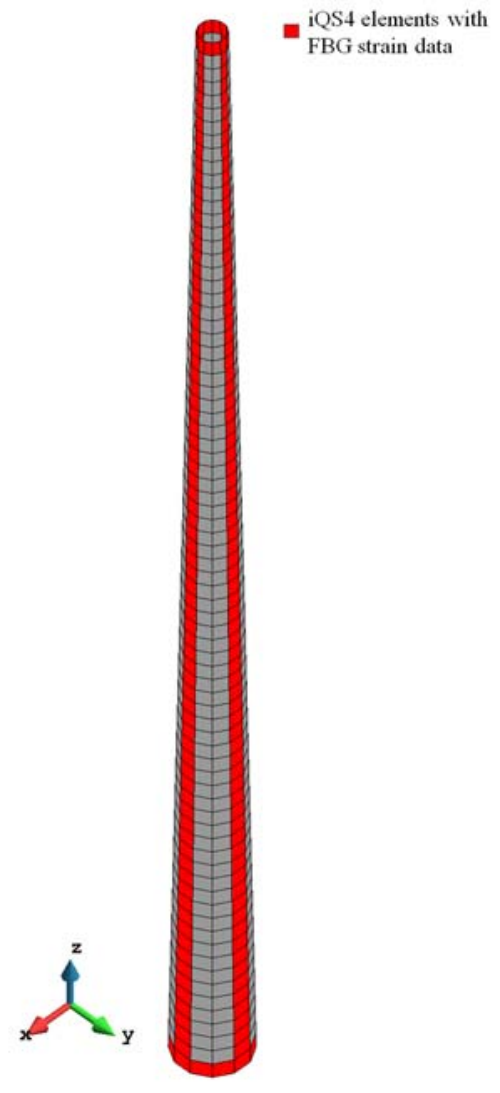

(a)

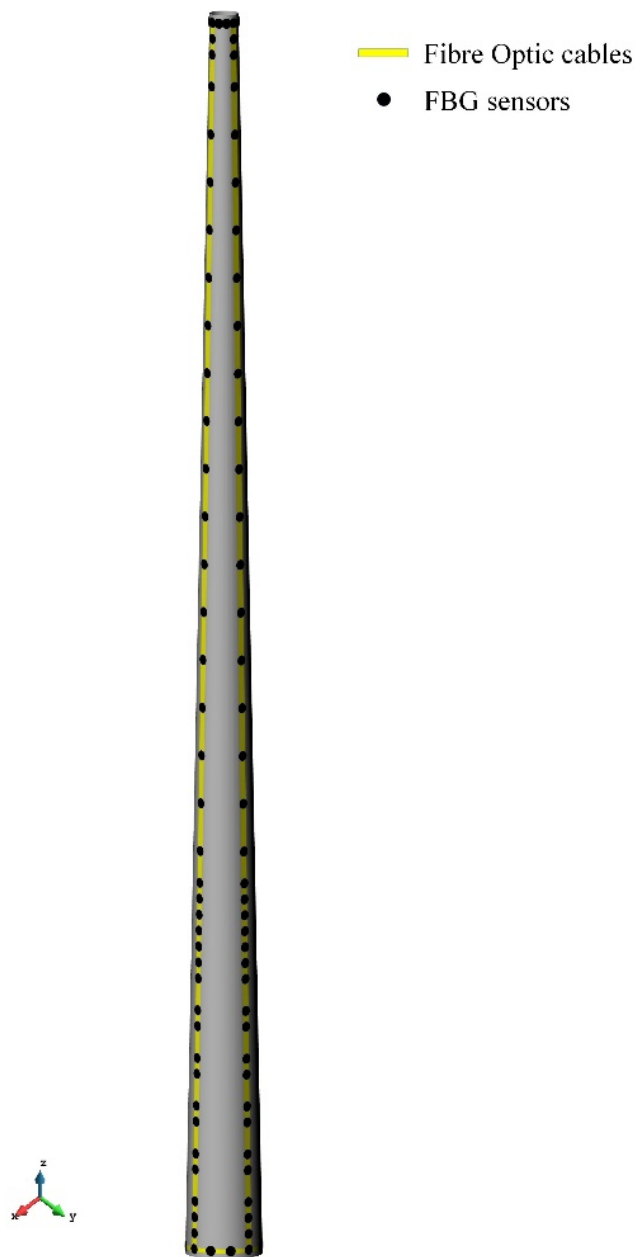

(b)

Figure 4. Sensor locations for reduced sensors case (a) 328 elements (b) 176 elements

\subsection{Static analysis}

For the static condition, iFEM analysis with full sensors and reduced sensors are performed. Maximum values of total displacements and von Mises stresses are calculated and listed in Table 1. The differences between iFEM/iFEM-R and FEM results are given in Table 2. According to the table, it can be seen that iFEM, both with full sensors or with reduced sensors, can predict the maximum value of the total displacements with high accuracy. The differences against the reference value are smaller than $1 \%$ for both cases. For the calculation of von Mises stresses, iFEM with full sensors obtains a value of $6.298 \mathrm{MPa}$ which is $1.368 \%$ higher than the reference value. With the reduction of the sensors, the extreme value is further over-estimated as $6.407 \mathrm{MPa}$ and the difference raises to $3.122 \%$. Even though the difference increases slightly due to the decrease of number of sensors, the percentage difference 
is still less than 5\%, which means the results of iFEM are very close to the reference FEM results. In addition, plots of the total displacements and von Mises stresses are also provided in Figs. 7 and 8. Since the tower only bears the weight of itself and top structures, it is expected that the displacements in z-direction will be the dominated one. The extreme value of the total displacements is concentrated at the top of the tower. The displacements have a tendency of increase from zero to the maximum value along with the height. As Figure 7 shows, the total displacement plots of the full-sensor and reduced-sensor iFEM cases are both indistinguishable with the reference FEM plot showing that the results of iFEM are in good agreement with FEM results. Concerning the von Mises stress, owing to the fact that the thickness of each section is fixed at $30 \mathrm{~mm}$ but the diameter of the hull is decreasing from the bottom to the top, the extreme von Mises stress occurs at the bottom boundary of the tower. At the same time, there are also some large stresses shown at the top of the tower. The minimum value of the von Mises stress is located around the middle of the tower. As a summary, the extreme values and plots of iFEM are pretty similar to reference FEM results which shows that in static condition iFEM, even using a low number of sensors, can provide accurate condition information of the tower.

Table 1. Results for static condition

\begin{tabular}{|c|c|c|}
\hline Static case & $U_{T}(\mathrm{~m})$ & $\sigma_{v m}(\mathrm{~Pa})$ \\
\hline a. FEM & $1.700 \mathrm{E}-03$ & $6.213 \mathrm{E}+06$ \\
\hline b. iFEM & $1.701 \mathrm{E}-03$ & $6.298 \mathrm{E}+06$ \\
\hline c. iFEM-R & $1.698 \mathrm{E}-03$ & $6.407 \mathrm{E}+06$ \\
\hline
\end{tabular}

Table 2. Differences between iFEM/iFEM-R and FEM results for static condition

\begin{tabular}{|c|c|c|}
\hline Differences & $U_{T}(\mathrm{~m})$ & $\sigma_{v m}(\mathrm{~Pa})$ \\
\hline FEM \& iFEM & $0.059 \%$ & $1.368 \%$ \\
\hline $\begin{array}{c}\text { FEM \& } \\
\text { iFEM-R }\end{array}$ & $0.118 \%$ & $3.122 \%$ \\
\hline
\end{tabular}




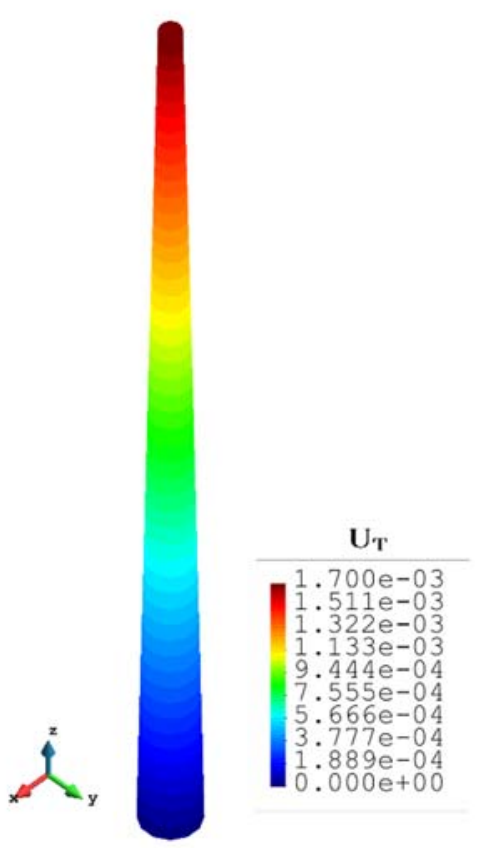

(a) FEM

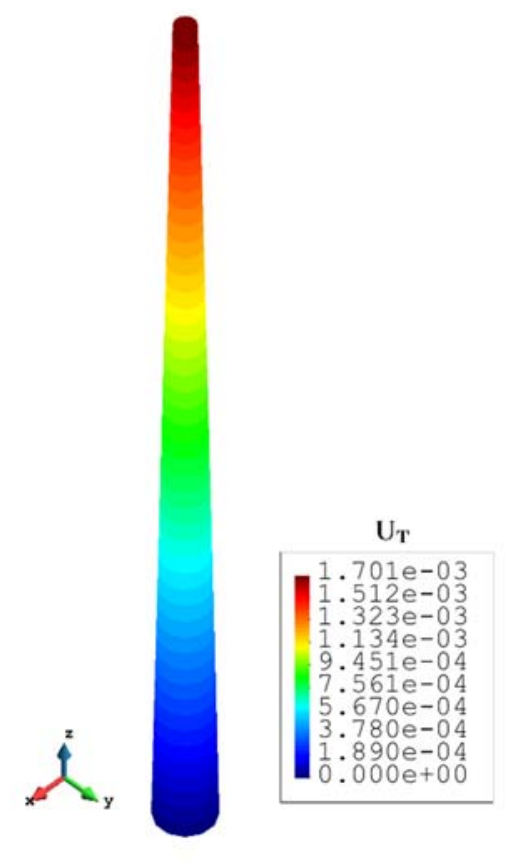

(b) iFEM

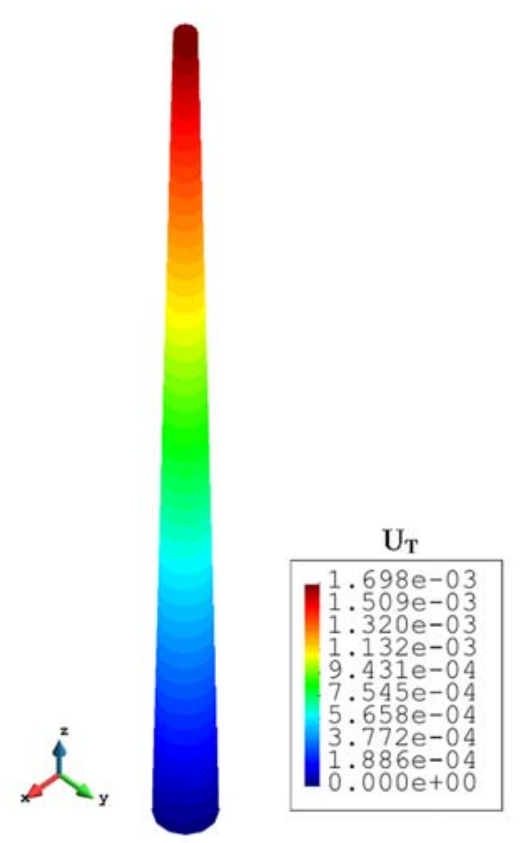

(c) iFEM-R

Figure 7. Contour plot of $U_{T}$ (unit: $\mathrm{m}$ ) for static condition

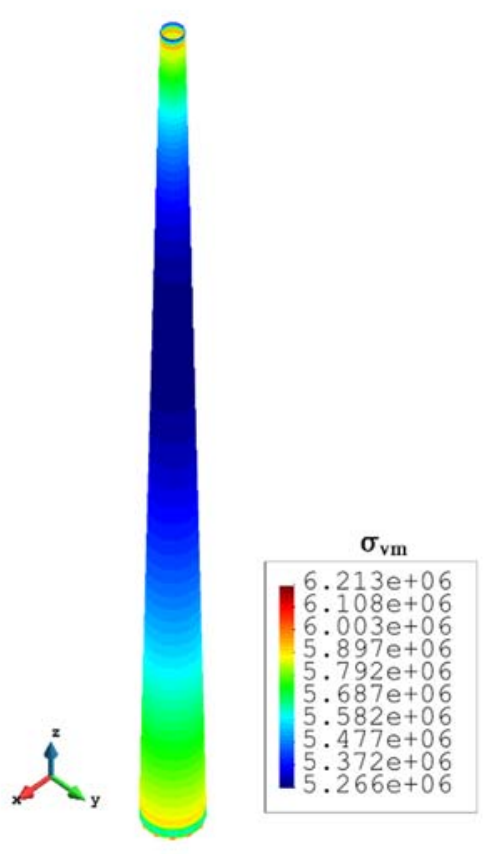

(a) FEM

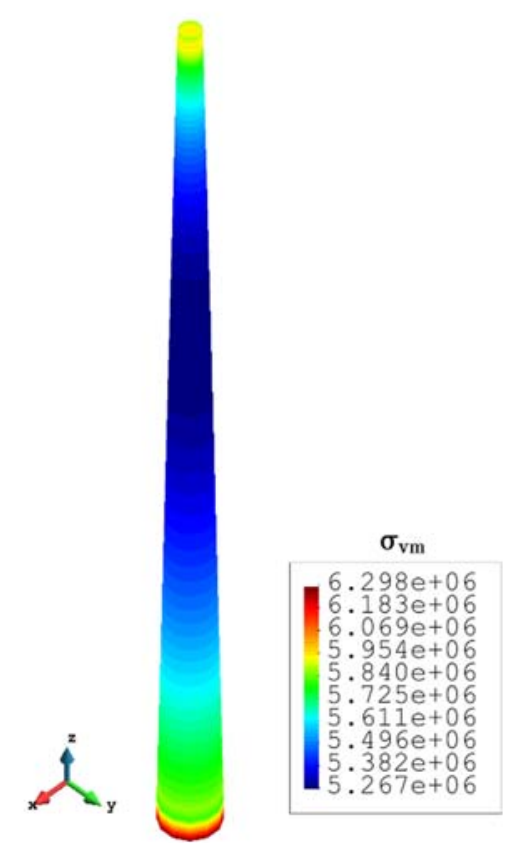

(b) iFEM

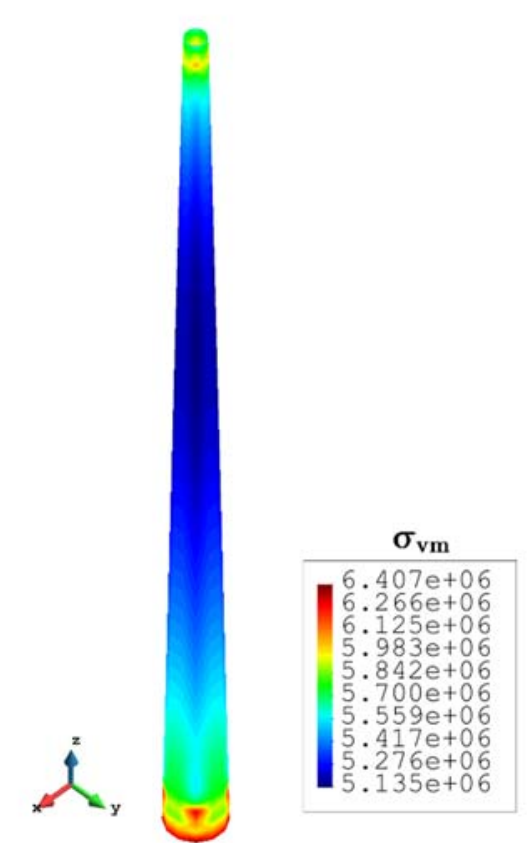

(c) iFEM-R

Figure 8. Contour plot of $\sigma_{v m}$ (unit: Pa) for static condition 


\subsection{Dynamic analysis}

For the health monitoring of the tower under dynamic loadings, aerodynamic and hydrodynamic forces are considered. The time duration of the analysis is $10 \mathrm{~s}$ and the total number of time steps is 200 which means that the time scale for each time step is $0.05 \mathrm{~s}$. The average wind speed is assumed to be $18 \mathrm{~m} / \mathrm{s}$ and the plot of the wind speed varying with time is given in Fig. 9.

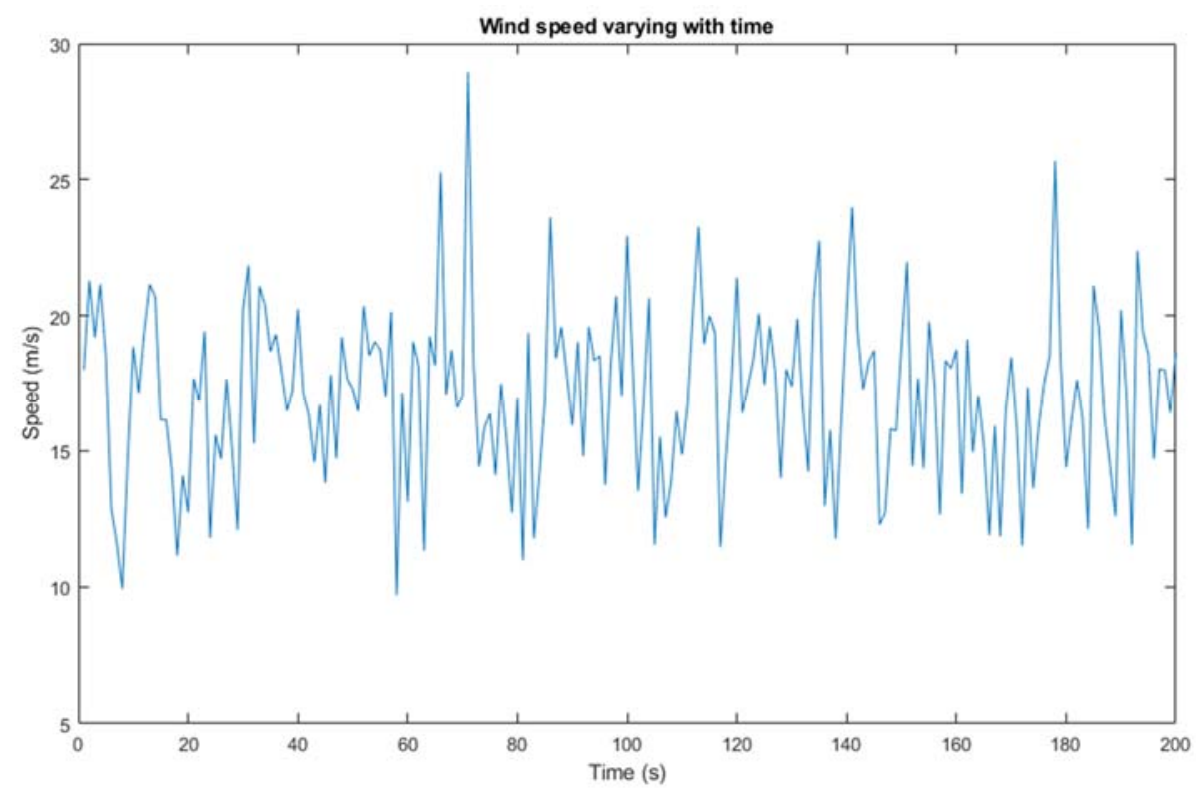

Figure 9. Variation of wind speed at reference height against time

The relationship between the wind velocity and the height is established by the following formula [38]:

$V_{\text {air }}=V_{r e f}\left(\frac{Z}{Z_{r}}\right)^{\alpha}$

where $V_{\text {air }}$ is the wind velocity at the corresponding position which has a height of $Z$.

$Z_{r}$ is the reference height, usually $10 \mathrm{~m}$ is preferred, $V_{r e f}$ is the wind velocity at the reference height, $\alpha$ is the Hellman coefficient and 0.12 is suggested for the natural air above the calm sea [38].

After calculating the wind velocity at different elevation, the aerodynamic loads can be obtained by a formula which is identical to the Morrison equation for hydrodynamic force calculation which is given as [36]:

$F_{\text {aero }}=\int_{0}^{h} \frac{1}{2} C_{\text {aero }} \rho_{\text {air }} A_{(z)} V_{\text {air }(z, t)}^{2} d z$ 
where $F_{\text {aero }}$ is the aerodynamic load at the height of $Z, C_{\text {aero }}$ is the aerodynamic coefficient which can be determined by the shape of the structure. For the current analysis, 0.5 is used because of the cylindrical shape. $\rho_{\text {air }}$ is the density of the wind and $1.25 \mathrm{~kg} / \mathrm{m}^{3}$ is used. $A_{(z)}$ is the exposed area of the structure at the height of $Z$. $V_{\text {air }}$ is the wind velocity calculated by using Eq. 7.

The wind forces acting on the tower are calculated section by section and applied as pressure to each section. The aerodynamic load on the blade of the OWT for the working condition can also be estimated by the same equation. The swept area of the blades is $624 \mathrm{~m}^{2}$ and this load is treated as a trust force and transformed to the top of the tower (the height of the RNA is selected at $45 \mathrm{~m}$ above the water surface for simplification). [36]

Morrison equation is used for the calculation of the hydrodynamic force. According to the Morrison equation, the hydrodynamic forces are formed by Drag force $\left(F_{D}\right)$ and Inertia force $\left(F_{I}\right)$. The detailed formula for these two forces when acting on the cylindrical structure is given as:

$$
\begin{aligned}
& F_{D}=\int_{-d}^{0} \frac{1}{2} \rho_{s e a} C_{d} A_{(z)} u_{(z, t)}\left|u_{(z, t)}\right| d z \\
& F_{I}=\int_{-d}^{0} \frac{1}{4} \pi \rho_{\text {sea }} C_{I} A_{(z)}^{2} u_{(z, t)}^{\prime} d z
\end{aligned}
$$

where $\rho_{\text {sea }}$ is the density of the seawater and $1025 \mathrm{~kg} / \mathrm{m}^{3}$ is used. The drag coefficient $\left(C_{d}\right)$ and inertia coefficient $\left(C_{I}\right)$ are 0.7 and 2.0, respectively, just as recommended by ISO 1990-1 [39]. $A_{(z)}$ is the area per unit height. $u_{(z, t)}$ and $u_{(z, t)}^{\prime}$ are the velocities and the accelerations of the water particles, separately. For the current analysis, linear wave theory is used and the information of the wave is given in Table 3: 
Table 3. Wave parameters

\begin{tabular}{|c|c|c|}
\hline Items & Values & Units \\
\hline Wave height $\left(W_{h}\right)$ & 1.8 & $\mathrm{~m}$ \\
\hline Wave length $\left(W_{l}\right)$ & 40 & $\mathrm{~m}$ \\
\hline Wave period $\left(W_{t}\right)$ & 5 & $\mathrm{~s}$ \\
\hline Water depth $\left(W_{d}\right)$ & 20 & $\mathrm{~m}$ \\
\hline
\end{tabular}

The ratio of water depth to wave height is 0.5 , which is properly selected as between deep water and middle water conditions. Then the velocity and acceleration of the water particles can be calculated as:

$u_{(z, t)}=\frac{\pi W_{h}}{W_{t}} \frac{\cosh \left(\frac{2 \pi}{W_{l}} Z\right)}{\sin h\left(\frac{2 \pi}{W_{l}} W_{d}\right)} \cos (\theta)$

$\dot{u}_{(z, t)}=\frac{2 \pi^{2} W_{h}}{W_{t}^{2}} \frac{\cosh \left(\frac{2 \pi}{W_{l}} Z\right)}{\sin h\left(\frac{2 \pi}{W_{l}} W_{d}\right)} \sin (\theta)$

where $Z$ is the water depth of the reference location. $\theta$ is the phase angle and it can be calculated based on the position and time as:

$\theta=\frac{2 \pi}{W_{l}} x-\frac{2 \pi}{W_{t}} t$

The wave forces are calculated from the bottom of the tower to the free water surface for simplification and they are also applied in the format of pressure to the tower. For the current analysis, the hydrodynamic and aerodynamic forces are in the same direction because it is the most severe loading condition which will lead to most serious deformations.

Apart from extra loadings, the model is kept to be the same as the static case, i.e. not only the parameters, but also the sensor locations are fixed for both conditions. Every 
$0.05 \mathrm{~s}$ the results of the $\mathrm{iFEM}$ analysis are recorded and then the total displacements and von Mises stresses are subsequently calculated for comparison. Six nodes at $40 \mathrm{~m}$ height (general height) are selected and the locations of the nodes are varying from the windward direction to the leeward direction (Figure 10). Besides, another three nodes, at the same position as node 6 (Figure 10(f)) but at different heights, are also chosen. The heights are $10 \mathrm{~m}$ (underwater), $20 \mathrm{~m}$ (free water surface), and $60 \mathrm{~m}$ (near top boundary).

The results of these nodes are collected and shown in Figs. 11-14. Figure 11 shows the plots of the total displacements for the nodes at different heights. First of all, iFEM with full sensors can obtain accurate results regardless of the heights of the nodes. The reference FEM and iFEM with full sensors results match perfectly with each other for the four diagrams. However, iFEM with reduced sensors (iFEM-R) makes less estimation about the total displacement for all four nodes. However, it can be seen that the differences are becoming smaller with the increase of the height of the nodes. It should also be mentioned that the total displacements at $60 \mathrm{~m}$ are much larger than the total displacements at $10 \mathrm{~m}$, i.e. the total displacements will increase with the height of the tower. The large deformations are given more attention because they can obviously show the health condition of the structure. Therefore, the errors in the total displacements at $10 \mathrm{~m}$ and $20 \mathrm{~m}$ are still reasonable. For the von Mises stress, iFEM can predict the von Mises stress accurately for the nodes at $40 \mathrm{~m}$ and $60 \mathrm{~m}$. Even if the values of the von Mises stress are slightly over-valued for some zones by iFEM with reduced sensors, for the majority parts they are still in good agreement with reference FEM results. For the node at $20 \mathrm{~m}$, the situation is becoming more severe. It is interesting to find that the results of iFEM with reduced sensors are even better than the iFEM with full sensors. The results are not always over-estimated for this node and during the first two seconds, they are slightly less than FEM results. Since this node is at the free water surface and the wave forces and wind forces are both acting on this region, the strains around the free surface will suffer sharp changes resulting in complex variation of results. But the main trend of the iFEM results is still the same with the FEM results. Due to the complexity of loading conditions, the maximum von Mises stress are located at the bottom of the tower for most of the cases. At $10 \mathrm{~m}$ height, the results of both full-sensor and reduced-sensor iFEM are bigger than the reference, but the differences between full-sensor condition and FEM 
are smaller than the reduced-sensor condition in general. The results of von Mises stress from iFEM can be very useful for failure judgement.

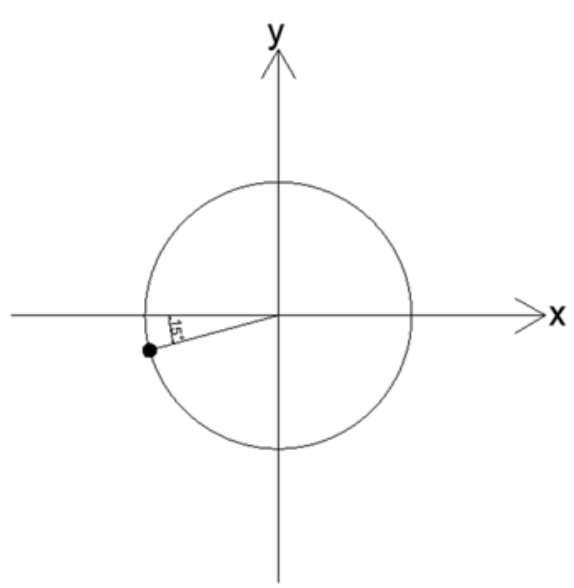

(a) node 1

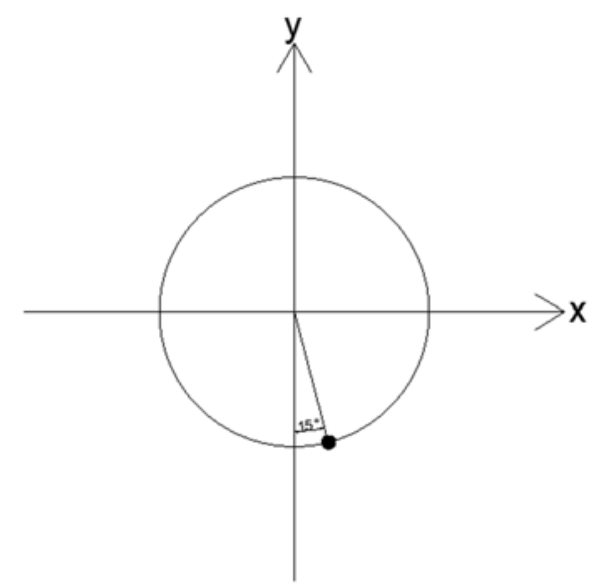

(d) node 4

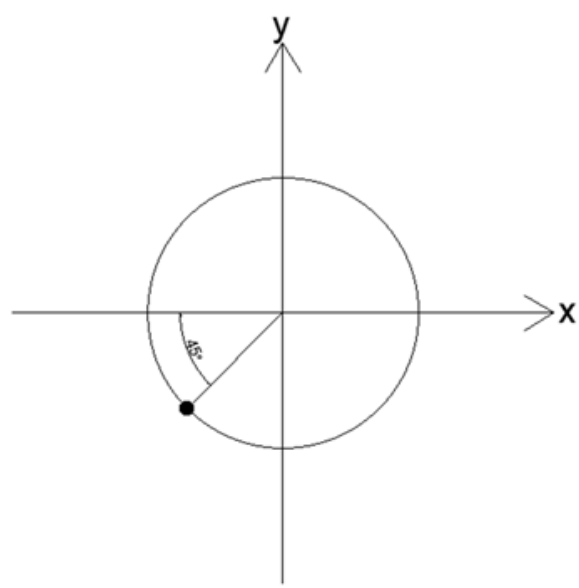

(b) node 2

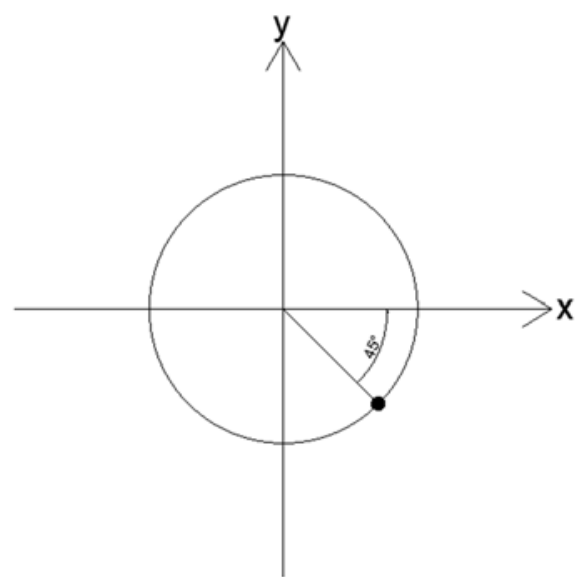

(e) node 5

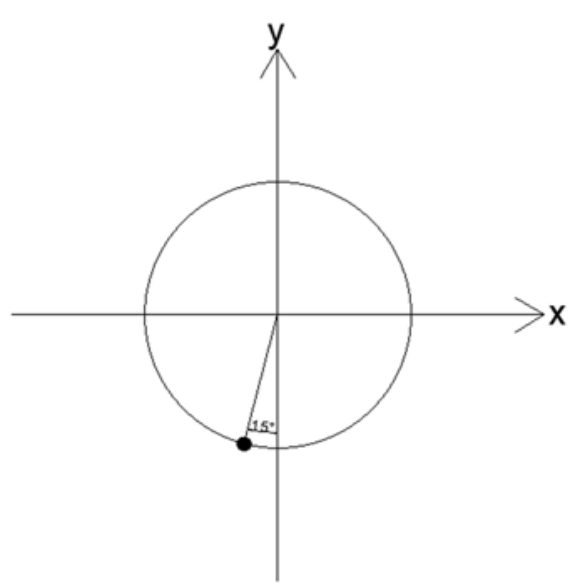

(c) node 3

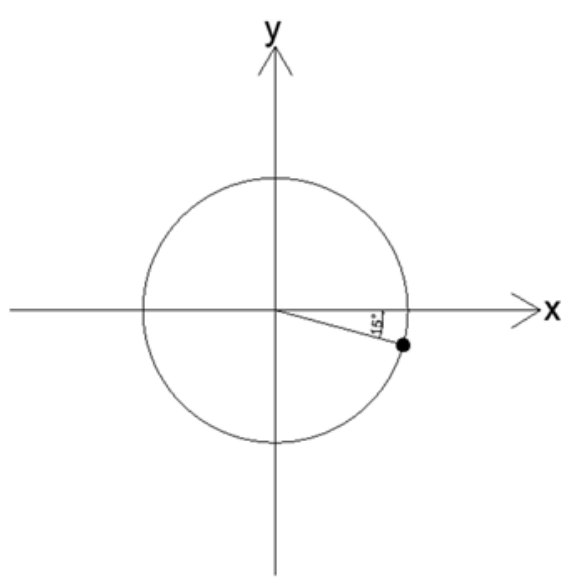

(f) node 6

Figure 10. The positions of the six nodes on the horizontal $x-y$ plane
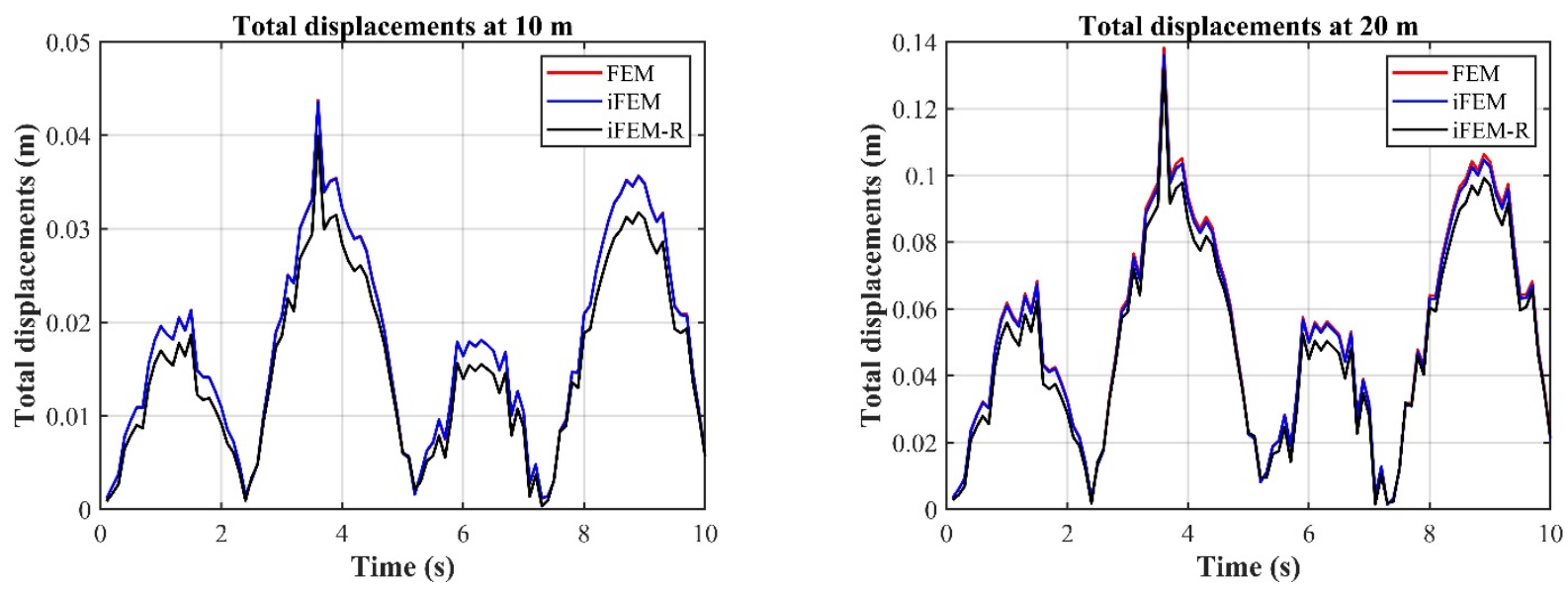

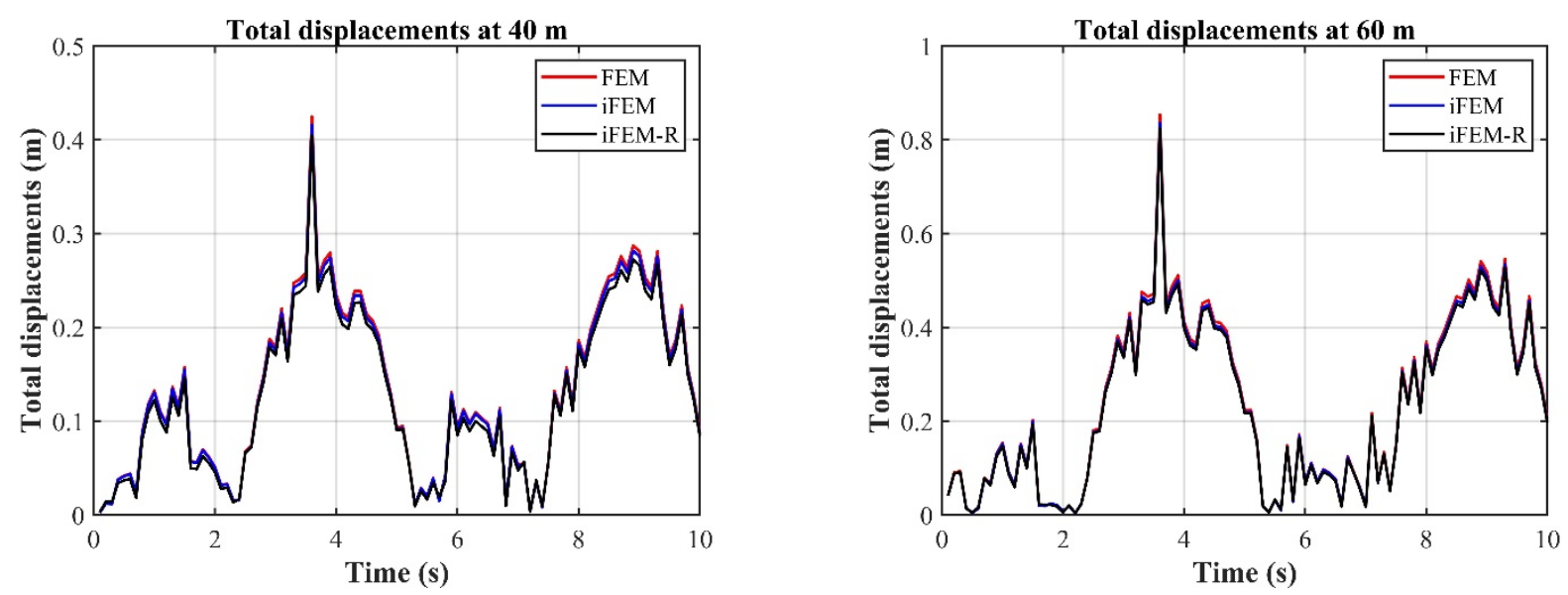

Figure 11. Variation of total displacements $U_{T}(\mathrm{~m})$ against time (s) for four nodes located at $10 \mathrm{~m}$, $20 \mathrm{~m}, 40 \mathrm{~m}$, and $60 \mathrm{~m}$.
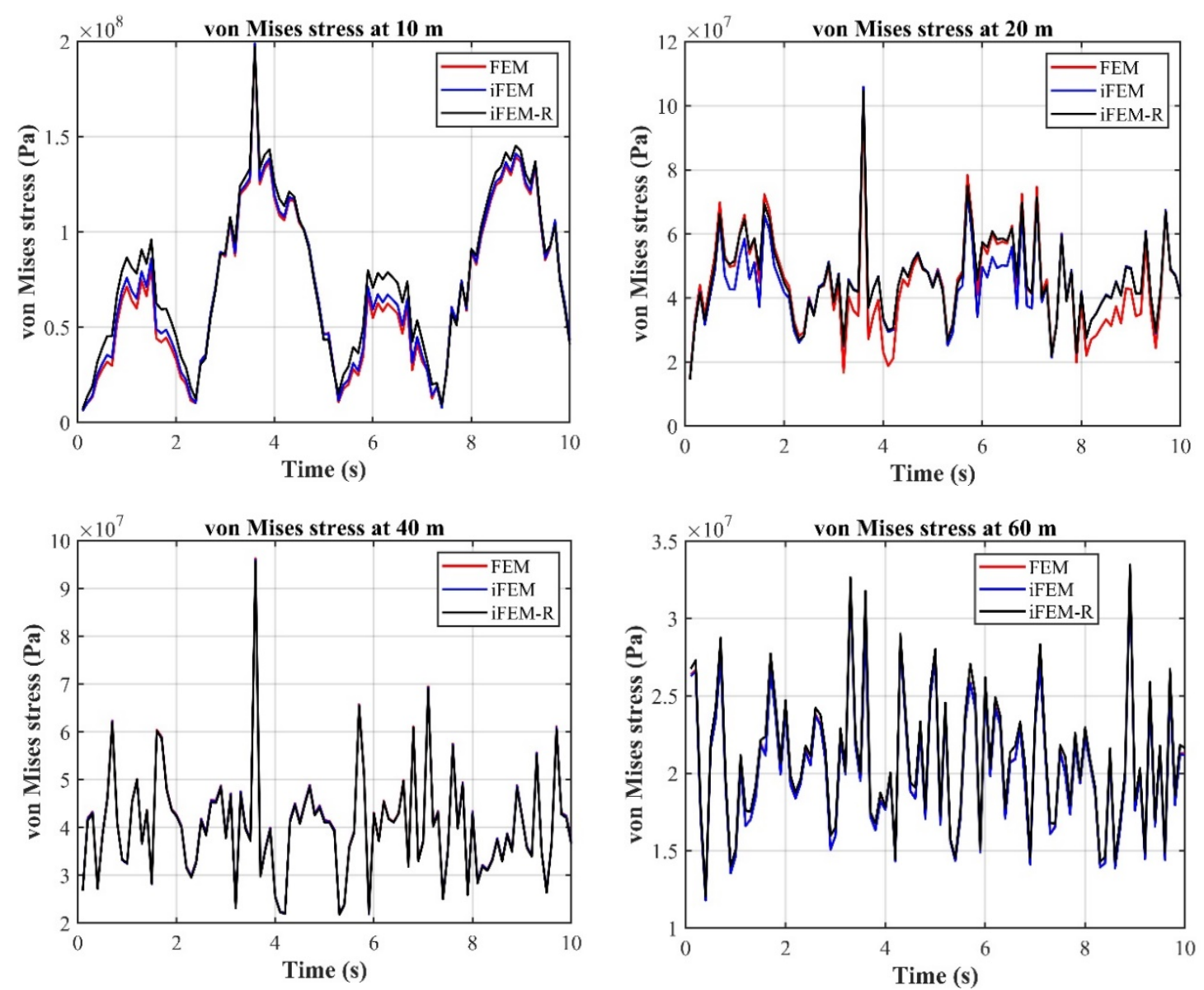

Figure 12. Variation of von Mises stresses $\sigma_{v m}(\mathrm{~Pa})$ against time (s) for four nodes located at $10 \mathrm{~m}, 20 \mathrm{~m}, 40 \mathrm{~m}$, and $60 \mathrm{~m}$. 
Six nodes in half of the ring lines at $40 \mathrm{~m}$ height are selected to investigate the effect of the nodal position on the same horizontal plane. For the total displacements, except for the values of the total displacements that are slightly under-estimated, the differences of all six nodes against their FEM results cannot be distinguished. Therefore, the nodal position on the same horizontal plane does not have a big influence on the total displacement monitoring. The von Mises stress of all nodes can also be correctly monitored. However, the differences at node 3 are much bigger than the remaining nodes. Since this node is at a position where only one side is subjected to wind loads, there are large differences between both sides resulting in these errors. The maximum von Mises stress usually occurs in the x-direction which means that the values at this node are not as important as node 1 and node 6 , so the differences are acceptable.
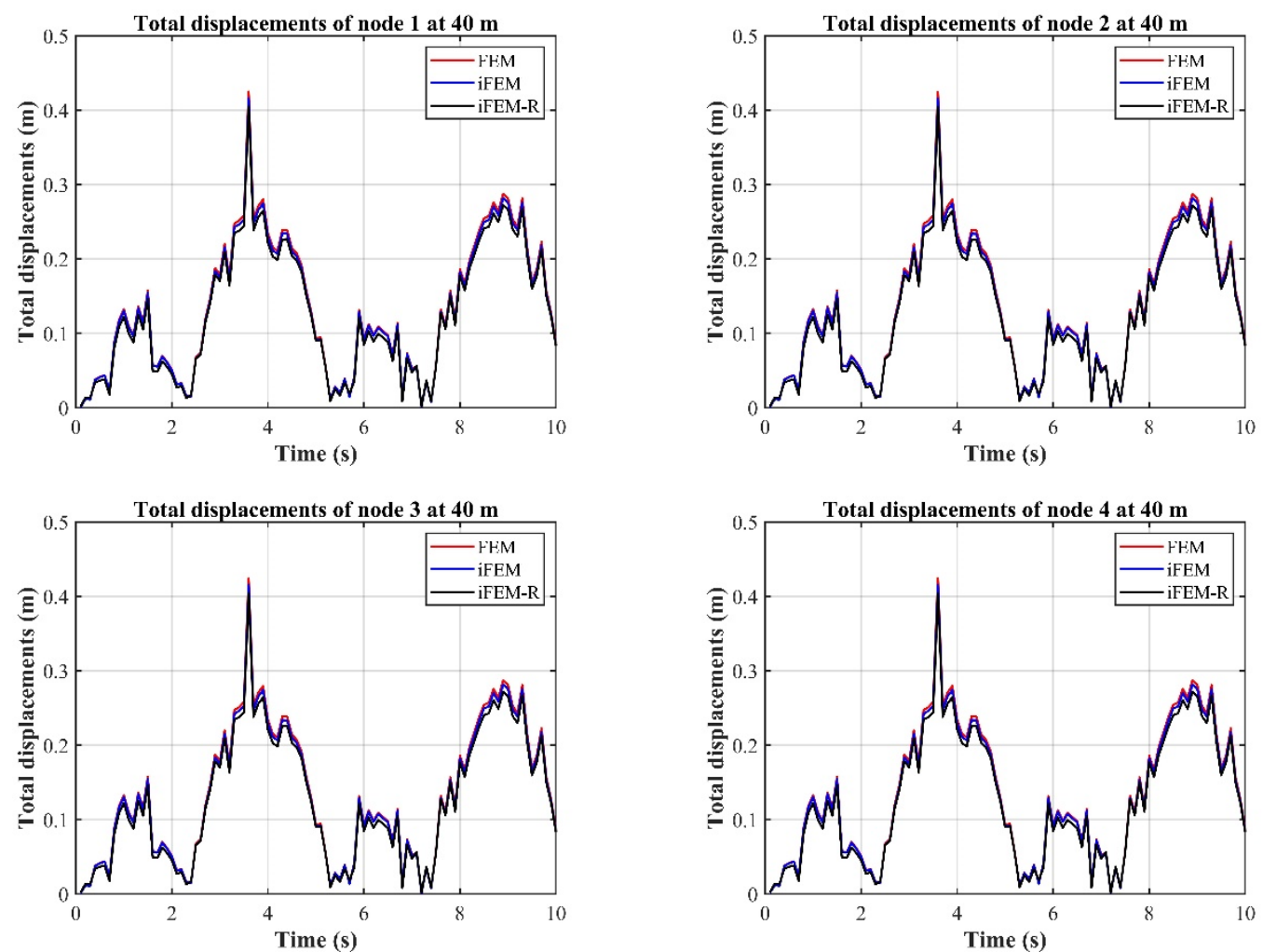

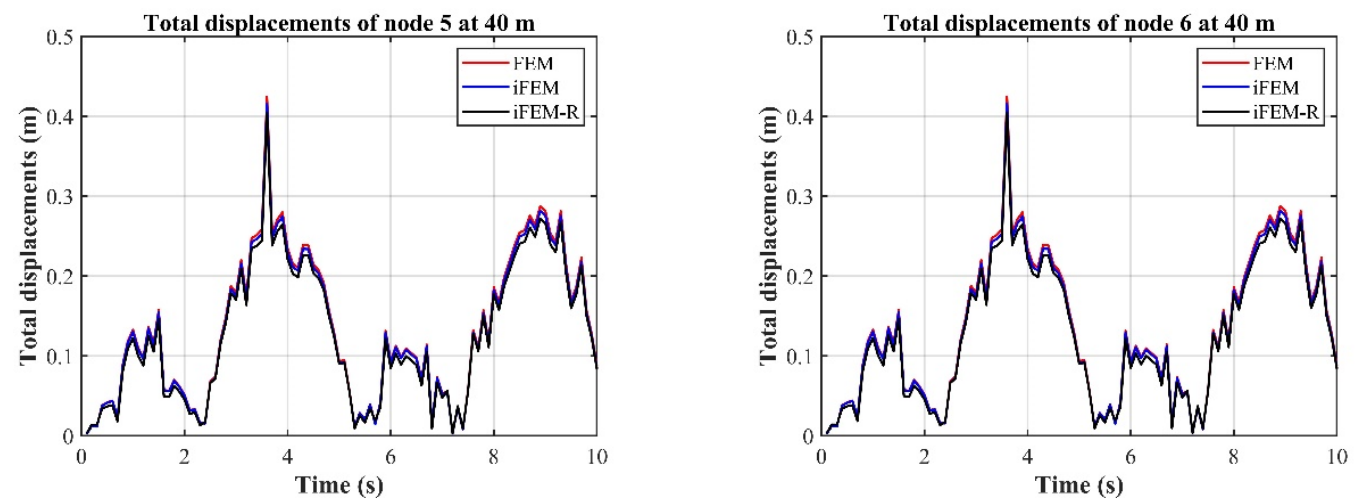

Figure 13. Variation of total displacements $U_{T}(\mathrm{~m})$ against time (s) for six nodes located at $40 \mathrm{~m}$.
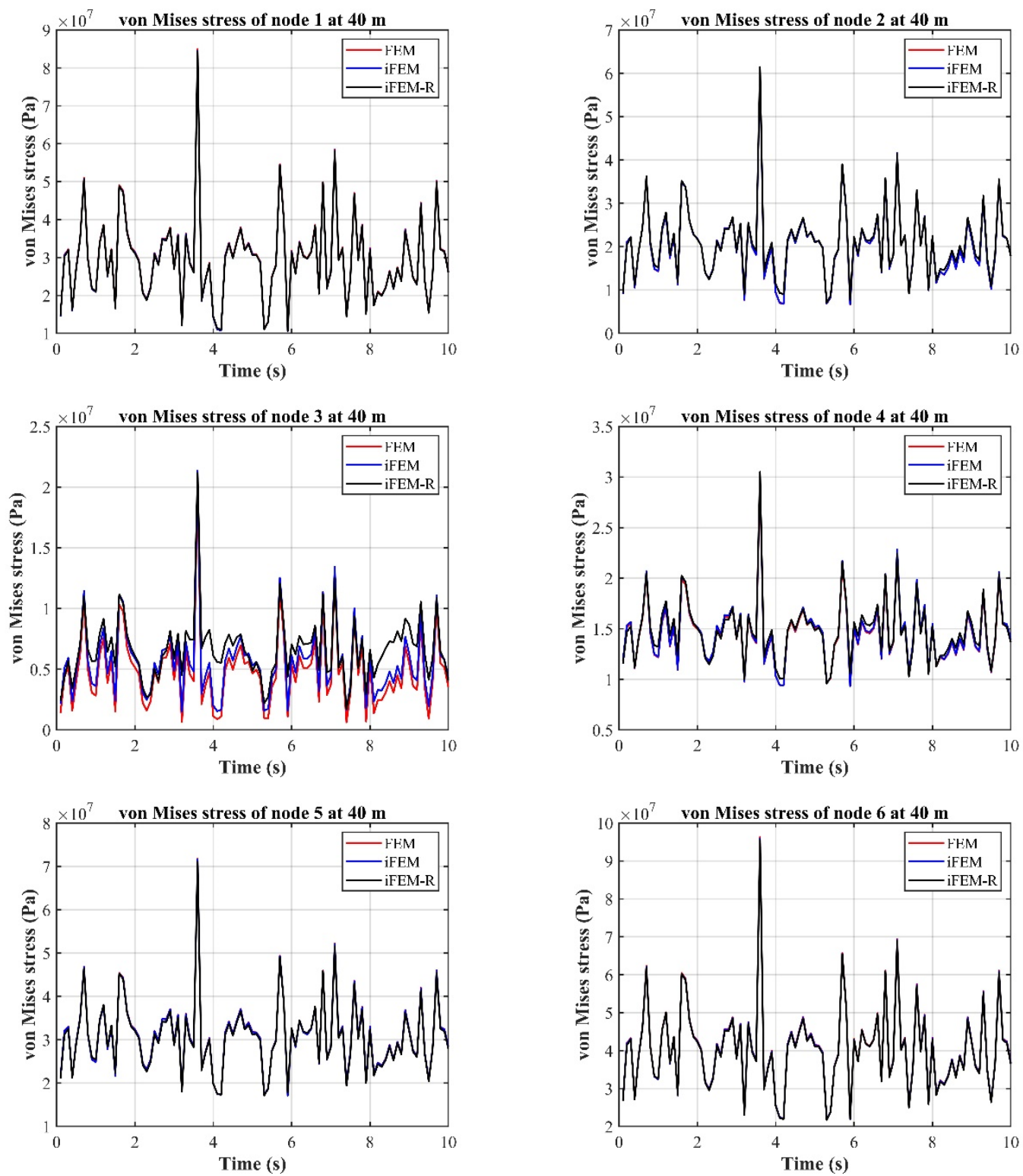

Figure 14. Variation of von Mises stresses $\sigma_{v m}(\mathrm{~Pa})$ against time (s) for six nodes located at $40 \mathrm{~m}$. 
Finally, the total displacements and von Mises stresses of the whole structure at $5 \mathrm{~s}$ are also used to evaluate the iFEM results. The extreme values are listed in Table 3 and the plots are also given as Figs. 15 and 16. In light of Table 3, iFEM whether with full sensors or reduced sensors can predict the correct extreme results with less than 5\% difference. The total displacements are also increasing from zero to the maximum value with the rise of the height but the dominated displacements are the deformations in the x-direction. The plots of the total displacements of iFEM are in line with the reference plot. The large stresses are concentrated at the bottom of the tower and the plots of iFEM can show this characteristic clearly. In conclusion, iFEM can perform accurate, reliable and robust SHM analysis for the tower of the OWT for both static and dynamic loading conditions. Currently used sensors, four longitudinal and two circumferential lines, would be enough for the monitoring process.

Table 4. Results of the dynamic condition at $5 \mathrm{~s}$

\begin{tabular}{|c|c|c|}
\hline Dynamic case (5s) & $U_{T}(\mathrm{~m})$ & $\sigma_{v m}(\mathrm{~Pa})$ \\
\hline a. FEM & $2.655 \mathrm{E}-01$ & $5.072 \mathrm{E}+07$ \\
\hline b. iFEM & $2.595 \mathrm{E}-01$ & $5.155 \mathrm{E}+07$ \\
\hline c. iFEM-R & $2.572 \mathrm{E}-01$ & $5.105 \mathrm{E}+07$ \\
\hline
\end{tabular}

Table 5. Differences between iFEM/iFEM-R and FEM results for dynamic condition at $5 \mathrm{~s}$

\begin{tabular}{|c|c|c|}
\hline Differences & $U_{T}(\mathrm{~m})$ & $\sigma_{v m}(\mathrm{~Pa})$ \\
\hline FEM \& iFEM & $2.260 \%$ & $1.636 \%$ \\
\hline FEM \& iFEM-R & $3.126 \%$ & $0.651 \%$ \\
\hline
\end{tabular}




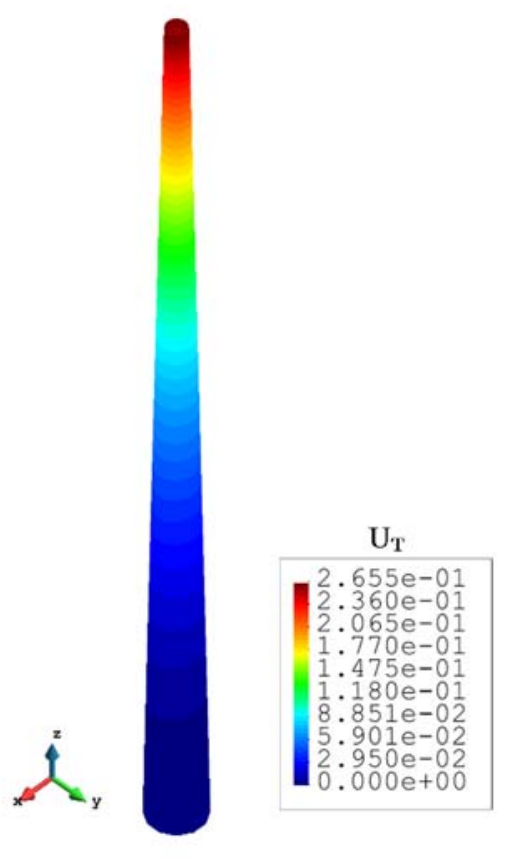

(a) FEM

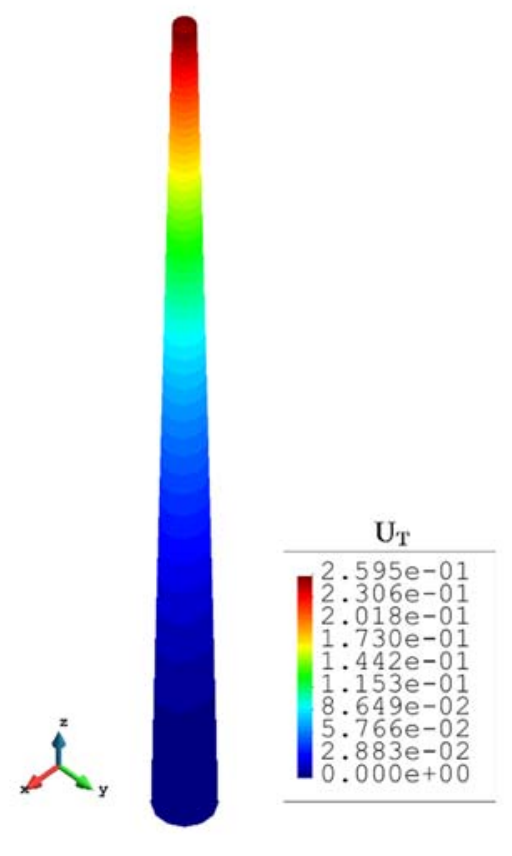

(b) iFEM

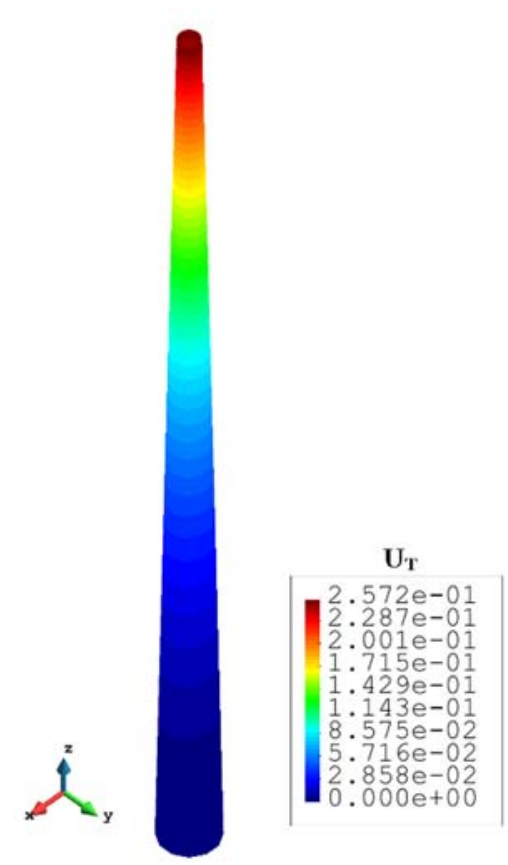

(c) iFEM-R

Figure 15. Contour plot of total displacements, $U_{T}$ (unit: $\mathrm{m}$ ) for the dynamic condition at $5 \mathrm{~s}$

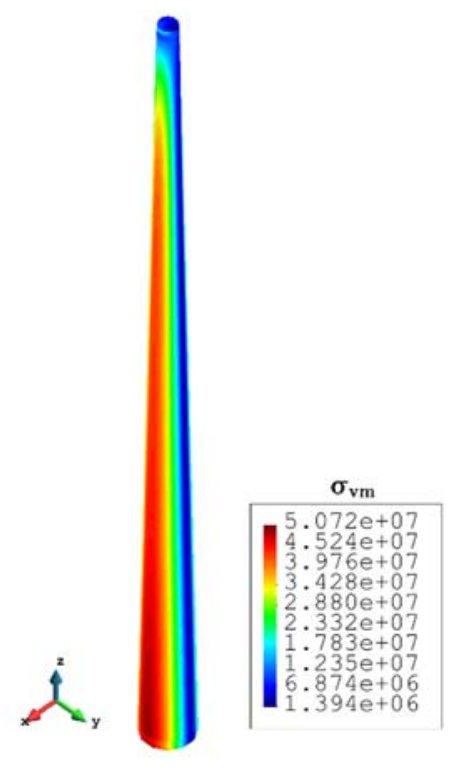

(a) FEM

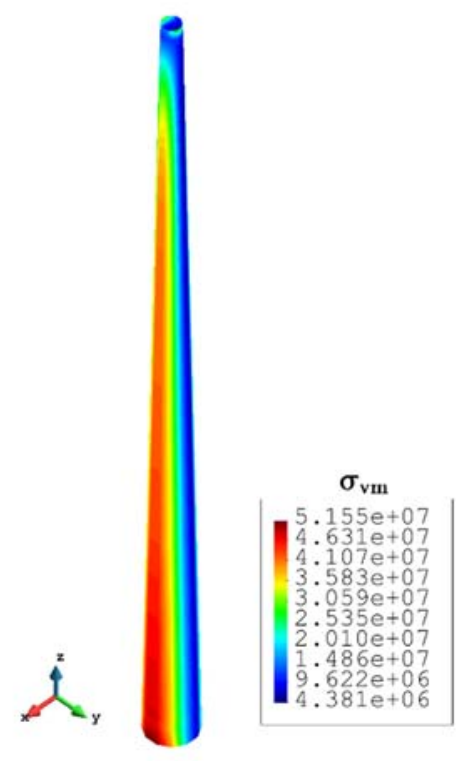

(b) iFEM

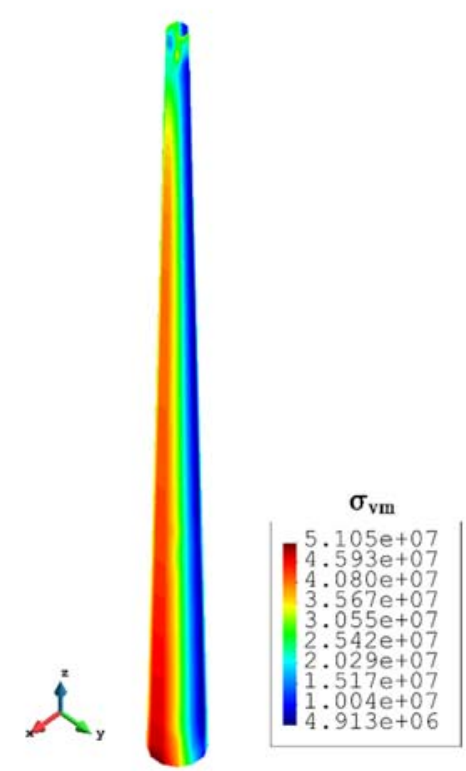

(c) iFEM-R

Figure 16. Contour plot of von Mises stresses, $\sigma_{v m}$ (unit: Pa) for the dynamic condition at $5 \mathrm{~s}$ 


\section{Conclusions}

In this paper, the iQS4 element is utilized for the iFEM analysis of the tower of OWTs. The tower is modeled with dense FEM and coarse iFEM meshes. The FEM analysis provides the "synthetic" strain data for the iQS4 element. Both static and dynamic loading conditions are considered. For the static condition, only the gravity of the upper structures and tower itself are taken into account whereas the aerodynamic and hydrodynamic forces are considered during the dynamic analysis. iFEM analysis is performed with both full sensors and reduced sensors. The practical number of sensors and their locations are explored. After determination of optimum sensor locations, the final sensor configuration includes four longitudinal and two ring cables. In these cables, the current discretized locations of the sensors would be sufficient for monitoring the tower under real loading conditions. It is also shown that the sections under aerodynamic loadings needs less number of sensors than the underwater sections. Total displacements and von Mises stresses are selected as the judging criteria for the accuracy of the iFEM analysis. For the dynamic analysis, different heights and different locations at the same height are selected for comparison purposes. After comparing the results and plots of the iFEM analysis with the reference FEM analysis, the iFEM-iQS4 element is proven to generate a SHM system with high accuracy for the tower of the OWTs. Future research can focus on other components of the OWTs to achieve a complete SHM system for entire OWTs based on iFEM.

\section{Acknowledgement}

Authors would like to thank Offshore Renewable Energy Catapult for the financial support for this study.

\section{References}

1. Tchakoua, P., Wamkeue, R., Ouhrouche, M., Slaoui-Hasnaoui, F., Tameghe, T.A. and Ekemb, G., 2014. Wind turbine condition monitoring: State-of-the-art review, new trends, and future challenges. Energies, 7(4), pp.2595-2630.

2. Ciang, C.C., Lee, J.R. and Bang, H.J., 2008. Structural health monitoring for a wind turbine system: a review of damage detection methods. Measurement science and technology, 19(12), p.122001. 
3. Mieloszyk, M. and Ostachowicz, W., 2017. An application of Structural Health Monitoring system based on FBG sensors to offshore wind turbine support structure model. Marine Structures, 51, pp.65-86.

4. Yang, W., Tavner, P.J., Crabtree, C.J., Feng, Y. and Qiu, Y., 2014. Wind turbine condition monitoring: technical and commercial challenges. Wind Energy, 17(5), pp.673-693.

5. Fu, Z. and Yuan, Y., 2012. Status and prospects on condition monitoring technologies of offshore wind turbine. Dianli Xitong Zidonghua(Automation of Electric Power Systems), 36(21), pp.121-129.

6. Lu, B., Li, Y., Wu, X. and Yang, Z., 2009, June. A review of recent advances in wind turbine condition monitoring and fault diagnosis. In 2009 IEEE Power Electronics and Machines in Wind Applications (pp. 1-7). IEEE.

7. Kefal, A., 2017. Structural health monitoring of marine structures by using inverse finite element method (Doctoral dissertation, University of Strathclyde).

8. Gherlone, M., Cerracchio, P., Mattone, M., Di Sciuva, M. and Tessler, A., 2012. Shape sensing of 3D frame structures using an inverse finite element method. International Journal of Solids and Structures, 49(22), pp.3100-3112.

9. Adams, D., White, J., Rumsey, M. and Farrar, C., 2011. Structural health monitoring of wind turbines: method and application to a HAWT. Wind Energy, 14(4), pp.603-623.

10. Weijtjens, W., Verbelen, T., De Sitter, G. and Devriendt, C., 2016. Foundation structural health monitoring of an offshore wind turbine - a full-scale case study. Structural Health Monitoring, 15(4), pp.389-402.

11. Currie, M., Saafi, M., Tachtatzis, C. and Quail, F., 2013. Structural health monitoring for wind turbine foundations. Proceedings of the ICE-Energy, 166(4), pp.162-169.

12. Yi, J.H., Park, J.S., Han, S.H. and Lee, K.S., 2013. Modal identification of a jacket-type offshore structure using dynamic tilt responses and investigation of tidal effects on modal properties. Engineering Structures, 49, pp.767-781.

13. Devriendt, C., Magalhães, F., Weijtjens, W., De Sitter, G., Cunha, Á. and Guillaume, P., 2014. Structural health monitoring of offshore wind turbines using automated operational modal analysis. Structural Health Monitoring, 13(6), pp.644-659.

14. Rolfes, R., Zerbst, S., Haake, G., Reetz, J. and Lynch, J.P., 2007, September. Integral SHM-system for offshore wind turbines using smart wireless sensors. In Proceedings of the 6th International Workshop on Structural Health Monitoring (pp. 11-13). DEStech Publications Inc. Stanford, CA, USA.

15. Schroeder, K., Ecke, W., Apitz, J., Lembke, E. and Lenschow, G., 2006. A fibre Bragg grating sensor system monitors operational load in a wind turbine rotor blade. Measurement Science and Technology, 17(5), p.1167.

16. Tessler, A. and Hughes, T.J., 1983. An improved treatment of transverse shear in the Mindlin-type four-node quadrilateral element. Computer methods in applied mechanics and engineering, 39(3), pp.311-335. 
17. Tessler, A. and Spangler, J.L., 2003. A variational principle for reconstruction of elastic deformations in shear deformable plates and shells. NASA/TM-2003212445 .

18. Tessler, A. and Spangler, J.L., 2004. Inverse FEM for full-field reconstruction of elastic deformations in shear deformable plates and shells. $2^{\text {nd }}$ European Workshop on Structural Health Monitoring, 7-9 July 2004, Munich, Germany.

19. Kefal, A., Hizir, O. and Oterkus, E., 2015, August. A smart system to determine sensor locations for structural health monitoring of ship structures. In Proceedings of the 9th International Workshop on Ship and Marine Hydrodynamics, Glasgow, UK (pp. 26-28).

20. Kefal, A., Oterkus, E., Tessler, A. and Spangler, J.L., 2016. A quadrilateral inverse-shell element with drilling degrees of freedom for shape sensing and structural health monitoring. Engineering science and technology, an international journal, 19(3), pp.1299-1313.

21. Gherlone, M., Cerracchio, P., Mattone, M., Di Sciuva, M. and Tessler, A., 2011. Beam shape sensing using inverse finite element method: theory and experimental validation. POLITECNICO DI TORINO (ITALY).

22. Gherlone, M., Cerracchio, P., Mattone, M., Di Sciuva, M. and Tessler, A., 2014. An inverse finite element method for beam shape sensing: theoretical framework and experimental validation. Smart Materials and Structures, 23(4), p.045027.

23. Gherlone, M., Cerracchio, P., Mattone, M., Di Sciuva, M. and Tessler, A., 2011. Dynamic shape reconstruction of three-dimensional frame structures using the inverse finite element method. NASA/TP-2011-217315.

24. Kefal, A., Tessler, A. and Oterkus, E., 2017. An enhanced inverse finite element method for displacement and stress monitoring of multilayered composite and sandwich structures. Composite Structures, 179, pp.514-540.

25. Kefal, A., 2019. An efficient curved inverse-shell element for shape sensing and structural health monitoring of cylindrical marine structures. Ocean Engineering, 188, p.106262.

26. Cerracchio, P., Gherlone, M., Di Sciuva, M. and Tessler, A., 2015. A novel approach for displacement and stress monitoring of sandwich structures based on the inverse Finite Element Method. Composite Structures, 127, pp.69-76.

27. Kefal, A. and Oterkus, E., 2016. Displacement and stress monitoring of a chemical tanker based on inverse finite element method. Ocean Engineering, 112, pp.33-46.

28. Kefal, A. and Oterkus, E., 2016. Displacement and stress monitoring of a Panamax containership using inverse finite element method. Ocean Engineering, 119, pp.16-29.

29. Kefal, A., Mayang, J.B., Oterkus, E. and Yildiz, M., 2018. Three dimensional shape and stress monitoring of bulk carriers based on iFEM methodology. Ocean Engineering, 147, pp.256-267.

30. Kefal, A. and Oterkus, E., 2017. Shape and stress sensing of offshore structures by using inverse finite element method. Progress in the Analysis and Design of Marine Structures; Guedes Soares, C., Garbatov, Y., Eds, pp.141-148. 
31. Colombo, L., Sbarufatti, C. and Giglio, M., 2019. Definition of a load adaptive baseline by inverse finite element method for structural damage identification. Mechanical Systems and Signal Processing, 120, pp.584-607.

32. Ren, L., Li, H., Zhou, J., Li, D.S. and Sun, L., 2006. Health monitoring system for offshore platform with fiber Bragg grating sensors. Optical Engineering, 45(8), p.084401.

33. Bang, H.J., Kim, H.I., Jang, M. and Han, J.H., 2012, February. Tower deflection monitoring of a wind turbine using an array of fiber Bragg grating sensors. In ASME 2011 Conference on Smart Materials, Adaptive Structures and Intelligent Systems (pp. 473-479). American Society of Mechanical Engineers Digital Collection.

34. Bang, H.J., Kim, H.I. and Lee, K.S., 2012. Measurement of strain and bending deflection of a wind turbine tower using arrayed FBG sensors. International journal of precision engineering and manufacturing, 13(12), pp.2121-2126.

35. Bang, H.J., Ko, S.W., Jang, M.S. and Kim, H.I., 2012, May. Shape estimation and health monitoring of wind turbine tower using a FBG sensor array. In 2012 IEEE International Instrumentation and Measurement Technology Conference Proceedings (pp. 496-500). IEEE.

36. Dagli, B.Y., Tuskan, Y. and Gökkuş, Ü., 2018. Evaluation of Offshore Wind Turbine Tower Dynamics with Numerical Analysis. Advances in Civil Engineering, 2018.

37. Gücüyen, E., 2017. Analysis of offshore wind turbine tower under environmental loads. Ships and Offshore Structures, 12(4), pp.513-520.

38. Van Der Tempel, J., 2006. Design of support structures for offshore wind turbines. $\mathrm{PhD}$ Thesis, Technical University of Delft.

39. ISO, P., 2005. natural gas industries. Specific requirements for offshore structures part 1: Metocean design and operating considerations. International Organization for Standardization, ISO, pp.19901-1. 